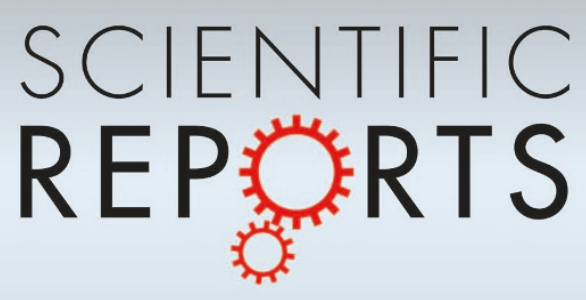

OPEN

SUBJECT AREAS:

PHYSICS

MATERIALS SCIENCE

APPLIED PHYSICS

CONDENSED-MATTER PHYSICS

Received

17 September 2012

Accepted

9 October 2012

Published

15 November 2012

Correspondence and requests for materials should be addressed to J.Z. (zhaoij@dlut.edu.

$\mathrm{cn})$

\section{Initial geometries, interaction mechanism and high stability of silicene on $\mathrm{Ag}(1111)$ surface}

\author{
Junfeng Gao \& Jijun Zhao
}

Laboratory of Materials Modification by Laser, lon and Electron Beams (Dalian University of Technology), Ministry of Education, Dalian 116024, China.

Using $a b$ initio methods, we have investigated the structures and stabilities of $\mathrm{Si}_{\mathrm{N}}$ clusters $(\mathrm{N} \leq 24)$ on $\mathrm{Ag}(111)$ surface as the initial stage of silicene growth. Unlike the dome-shaped graphene clusters, Si clusters prefer nearly flat structures with low buckling, more stable than directly deposition of the 3D freestanding Si clusters on Ag surface. The p-d hybridization between Ag and $\mathrm{Si}$ is revealed as well as $\mathrm{sp}^{2}$ characteristics in $\mathrm{Si}_{\mathrm{N}} @ \mathrm{Ag}(111)$. Three types of silicene superstructures on $\mathrm{Ag}(111)$ surface have been considered and the simulated STM images are compared with experimental observations. Molecular dynamic simulations show high thermal stability of silicene on $\mathrm{Ag}(111)$ surfaces, contrast to that on $\mathrm{Rh}(111)$. The present theoretical results constitute a comprehensive picture about the interaction mechanism of silicene on $\mathrm{Ag}(111)$ surface and explain the superiority of Ag substrate for silicene growth, which would be helpful for improving the experimentally epitaxial growth of silicene.

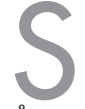

timulated by the graphene boom, silicon monolayer (ML) with honeycomb geometry, namely, silicene, has been theoretically predicted ${ }^{1-5}$ and experimentally synthesized ${ }^{6-15}$. Similar to its graphene counterpart, freestanding silicene sheet is also composed of hexagonal rings but with a slight buckling of about 0.44 $\AA$ A according to density functional theory (DFT) calculations ${ }^{2}$. Very excitingly, the band structures of low-buckled silicene resemble those of graphene, that is, $\pi$ and $\pi^{*}$ bands cross linearly at the Fermi level of the Brillouin zone, forming the so-called "Dirac cones"1,2,16-18. Hence, the charge carriers in silicene behave like Dirac massless fermions with ultrahigh Fermi velocity of the magnitude of $10^{5} \sim 10^{6} \mathrm{~m} / \mathrm{s}^{1,2,13,19,20}$. Besides, it was recently demonstrated that silicene with topologically nontrivial electronic structures can realize the quantum spin Hall effect in an experimentally accessible low temperature regime ${ }^{21}$. Though pristine silicene is a semimetal, tunable band gap in silicene monolayer can be opened up at the Fermi level by either applying external electric field ${ }^{22-25}$ or chemical functionalization with hydrogen ${ }^{26-29}$ and halogen elements ${ }^{29,30}$. In addition to the infinite silicene sheet, fascinating electronic and magnetic properties for the silicene nanoribbons have been predicted by first-principles calculations ${ }^{16}$. With these outstanding electronic and transport properties, integration of silicene into microelectronic devices is rather tempting since it is compatible with the mature silicon-based semiconductor technology.

Experimentally, silicene in the form of nanoribbons has been synthesized on $\operatorname{Ag}(001)^{6}$ and $\operatorname{Ag}(110)^{7-10}$ surfaces, respectively. Especially, the silicene nanoribbons on $\operatorname{Ag}(110)$ surface presented a magic width of $1.6 \mathrm{~nm}$ and aligned parallelly with each other in a well distributed way ${ }^{7-10}$. Reflection electron energy loss spectroscopy (REELS) measurement attested $\mathrm{sp}^{2}$-like hybridization of valence orbitals in the silicene nanoribbons ${ }^{10}$, whereas graphene-like band dispersion in the silicene nanoribbons has been testified by angle-resolved photoelectron spectra (ARPES) ${ }^{8}$. Moreover, these silicene nanoribbons on $\operatorname{Ag}(110)$ substrate show strong resistance towards oxidation $^{10}$.

Beyond the nanoribbons, Vogt et al. have epitaxially synthesized large area silicon monolayer on $\operatorname{Ag}(111)$ surface $^{13}$, making the theoretical predicted silicene ML comes true. With ARPES measurement, they demonstrated that the Dirac cone, which is the most intrinsic property of silicene distinguishing from common silicon, exists in the epitaxially silicene on $\mathrm{Ag}(111)$. Besides, compelling evidences for silicene honeycomb lattice, such as the Si $2 \mathrm{p}$ and Ag $4 \mathrm{~d}$ core level emission, the STM images, and the LEED pattern, were provided. Based on the experimental observations and DFT calculations, they proposed a superstructure of $(3 \times 3)$ silicene on $(4 \times 4)$ $\operatorname{Ag}(111)$ surface (which is usually called $\mathrm{Ag}(111)-(1 \times 1)$ lattice).

In addition, several other groups also synthesized silicene monolayer on $\operatorname{Ag}(111)$ surface and revealed the $(4 \times 4)$ superstructure, separately ${ }^{11,12,14}$. Wu's group observed one-atom-thick silicene sheet across the step edges 
of the $\operatorname{Ag}(111)$ surface without losing continuity of the atomic lattice $^{12}$, and they further proved the existence of massless Dirac fermions in silicene@ $\operatorname{Ag}(111)^{20}$. Besides, several other probable superstructures of silicene monolayer on $\operatorname{Ag}(111)$ surface were also proposed, such as $(\sqrt{13} \times \sqrt{13}) R 13.9^{\circ 11}$ and $(2 \sqrt{3} \times 2 \sqrt{3}) R 30^{\circ 14}$, implying that the interaction mechanism of silicene on $\operatorname{Ag}(111)$ surface is rather complicated and still unclear. Except for the $\operatorname{Ag}(111)$ substrate, Fleurence et al. obtained epitaxial silicene ML through surface segregation on the $\mathrm{ZrB}_{2}$ thin films grown on Si wafers ${ }^{15}$. The successful synthesis of silicene ML opens a new opportunity towards many potential applications, such as field effect transistor, ultrasensitive chemical sensors, and solar cells ${ }^{31,32}$.

A prerequisite for future utilization of silicene-based materials and devices is the mass production of silicene of high-quality. Clearly, elucidating the growth mechanism of silicene is imperative for the rapidly growing silicene research. Indeed, the growth behaviors of graphene on various transition metal (TM) substrates have been recently explored using $a b$ initio calculations ${ }^{33-39}$ and molecular dynamics (MD) simulations ${ }^{40,41}$. The behaviors of metal supported carbon clusters in the very initial stage of graphene growth, such as the ground state structures and the critical size for $\mathrm{sp}$ to $\mathrm{sp}^{2}$ transition (i.e., from linear chain to $2 \mathrm{D}$ island), were found to be quite different from those of freestanding carbon clusters ${ }^{33,34}$. On metal surfaces, graphene nanoclusters form dome-shaped islands spontaneously because the carbon-metal interaction mainly occurs at the edge atoms of the graphene patches ${ }^{35}$. In addition, the metal surfaces have significant influence on the diffusion and dissolution behaviors of carbon atoms ${ }^{40,42}$, as well as the edge reconstruction of graphene $\mathrm{e}^{38}$. Unlike carbon preferring $\mathrm{sp}^{2}$ bonding, silicon favors $\mathrm{sp}^{3}$ hybridization and there is no graphite-like $\mathrm{sp}^{2}$ allotrope for silicon solids. Intuitively, there must be some differences in the growth behaviors of silicene and graphene on the metal substrates. Hence, exploring the structures and stabilities of the TM-supported silicene clusters (as the early stage of growth) and silicene ML of different superstructures is of key importance. However, to the best of our knowledge, no such study was reported yet.

In this paper, we systematically investigate the interaction mechanism and stability of silicene clusters and monolayers on $\operatorname{Ag}(111)$ surface using $a b$ initio calculations. We show that the energetically unfavorable two-dimenstional (2D) honeycomb silicon clusters (up to 24 atoms) in vacuum can be stabilized by Ag substrate. Due to hybridization between silicene cluster and metal surface, no domeshaped silicon island is found on $\operatorname{Ag}(111)$ surface, in contrast to the metal-supported carbon clusters. The buckling of small silicene clusters on $\mathrm{Ag}(111)$ surface is lower than periodic silicene sheet either in vacuum or on $\operatorname{Ag}(111)$ surface. Analysis of electronic structures demonstrates that the $2 \mathrm{D} \mathrm{Si}_{24}$ cluster on $\mathrm{Ag}(111)$ surface retain certain $\mathrm{sp}^{2}$ character. Ab initio molecular dynamics (AIMD) simulations at $500 \mathrm{~K}$ confirm high stability of silicene ML on $\mathrm{Ag}(111)$ surface, but not on $\mathrm{Rh}(111)$ surface. Our theoretical results not only illustrate why $\operatorname{Ag}(111)$ surface is superior for epitaxial growth of silicene but also provide some useful insights into the synthesis of high-quality silicene on other probable metal surfaces.

\section{Results}

To characterize the stability of a silicon cluster on metal substrate, its formation energy is defined as:

$$
\varepsilon_{\mathrm{F}}=\left(\varepsilon_{\mathrm{T}}-N \times \varepsilon_{\mathrm{Si}}-\varepsilon_{\mathrm{Sub}}\right) / N
$$

where $\varepsilon_{\mathrm{T}}$ is the total energy for the silicon cluster and the metal substrate, $N$ is the number of atoms in the silicon cluster, $\varepsilon_{\mathrm{Si}}$ is the energy per atom of silicon solid with diamond lattice, $\varepsilon_{\text {Sub }}$ is the energy of the substrate. In the case of freestanding clusters in vacuum, $\varepsilon_{\mathrm{T}}$ becomes the total energy for the silicon cluster only, and the $\varepsilon_{\text {Sub }}$ term vanishes.

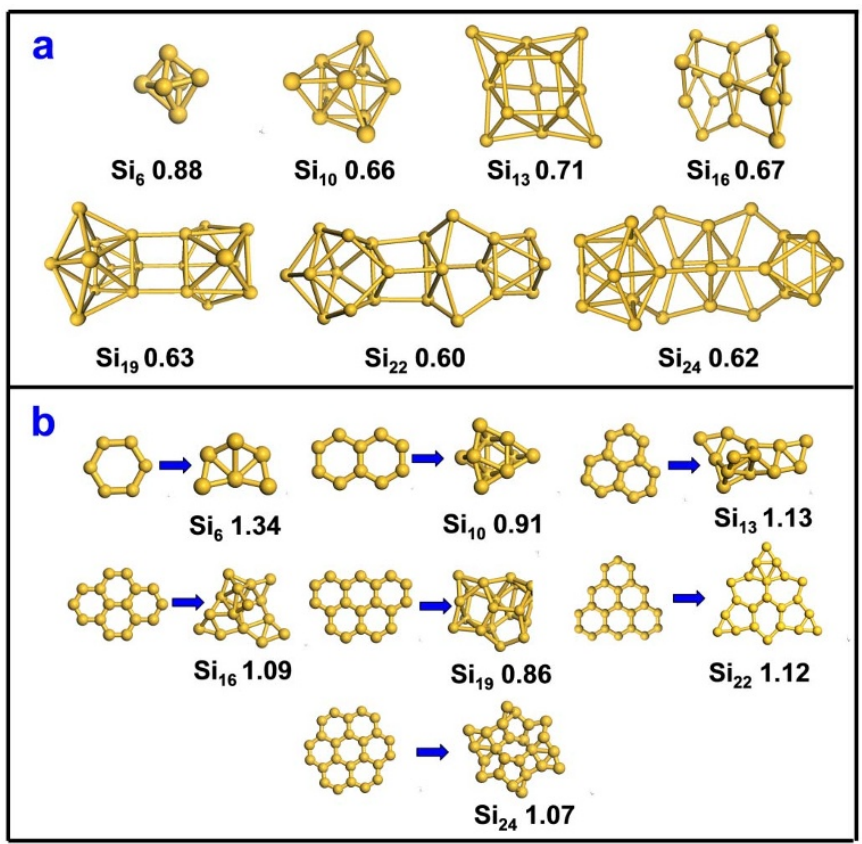

Figure $1 \mid$ (a) Most stable structures and their formation energies (eV per atom) for $\mathrm{Si}_{\mathrm{N}}$ clusters $(\mathrm{N}=6,10,13,16,19,22,24)$ in vacuum; (b) planar $\mathrm{Si}_{\mathrm{N}}$ clusters constructed by hexagonal rings (left) and their spontaneous transformation into severely distorted structures (right) after relaxation in vacuum.

Structures of $\mathrm{Si}_{\mathrm{N}}$ clusters in vacuum and on $\mathrm{Ag}(111)$ surface. Previously, Zeng's group has extensively explored the lowest-energy structures of freestanding $\mathrm{Si}_{\mathrm{N}}$ clusters $(\mathrm{N} \leq 30)^{43-45}$. Smaller $\mathrm{Si}_{6}$ and $\mathrm{Si}_{10}$ with sphere-like octahedron and tetracapped trigonal prism configurations are the magic clusters with exceptional stability ${ }^{43}$. The most stable configurations for the larger $\mathrm{Si}_{\mathrm{N}}$ clusters can be then obtained from the small clusters (like $\mathrm{Si}_{6}$ and $\mathrm{Si}_{10}$ ) as building units by adding atoms, fusing two clusters ${ }^{44}$, or assembling two clusters via some bridge atoms ${ }^{45}$. These ground state (abbreviated as GS in the following) structures are adopted here and their geometries and formation energies are presented in Fig. 1a. To examine the stability of $\mathrm{sp}^{2}$ silicene fragments without TM substrate, we constructed a series of $2 \mathrm{D} \mathrm{Si}_{\mathrm{N}}$ clusters $(\mathrm{N}=6,10,13,16,19,22,24)$ as aggregates of sixmembered rings (6MRs), which can be viewed as embryos of silicene ML. However, none of them are even metastable on the potential energy surface. After relaxation, these planar $\mathrm{Si}_{\mathrm{N}}$ clusters transform into severely buckled configurations spontaneously (see Fig. 1b). In vacuum, these highly distorted $\mathrm{Si}_{\mathrm{N}}$ clusters are less stable than the lowest-energy GS structures, with formation energy difference of about $0.25 \sim 0.52 \mathrm{eV} /$ atom.

For the purpose of comparison, we have explored selected freestanding planar $\mathrm{C}_{\mathrm{N}}$ clusters with the same 6MR-based configurations $^{46}$. The detailed results are given in Supplementary Fig. S1 of the Supporting Information (SI) online. Except for $\mathrm{C}_{10}$, which transforms from a double-hexagon configuration into a ten-membered ring after relaxation, the hexagon rings in all other freestanding $\mathrm{C}_{\mathrm{N}}$ clusters are well preserved upon optimization, even though they may not be the ground state configurations for these cluster sizes. The distinct difference between $\mathrm{Si}$ and $\mathrm{C}$ clusters suggests that the freestanding silicon clusters can not form stable $\mathrm{sp}^{2}$ structures spontaneously. However, low buckled 2D silicene sheets have been synthesized on $\operatorname{Ag}(111)$ surface in experiments ${ }^{11-14}$. Clearly, $\operatorname{Ag}(111)$ surface must be responsible for the stability of these $2 \mathrm{D}$ silicene structures and thus significantly affect the nucleation and growth behaviors of silicene clusters.

Fig. 2 depicts the equilibrium geometries and formation energies of silicon monomer, dimer and 6MR-based silicene-like clusters on 


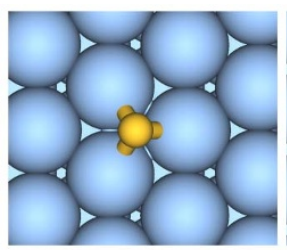

$\mathrm{Si}_{1} \mathbf{1 . 2 2}$

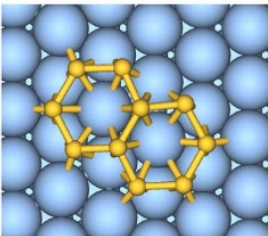

$\mathrm{Si}_{10} 0.53$

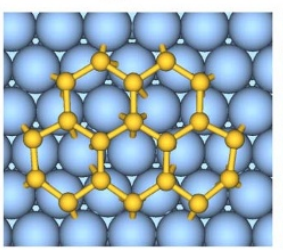

$\mathrm{Si}_{19} 0.42$

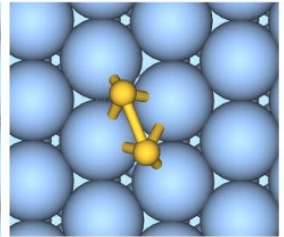

$\mathrm{Si}_{2} \mathbf{0 . 8 4}$

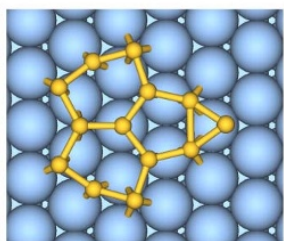

$\mathrm{Si}_{13} 0.49$

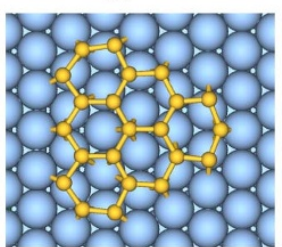

$\mathrm{Si}_{22} \mathbf{0 . 4 2}$

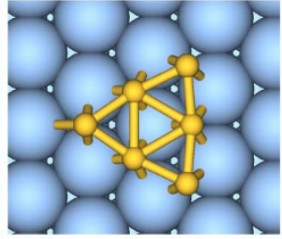

$\mathrm{Si}_{6} \mathbf{0 . 5 0}$

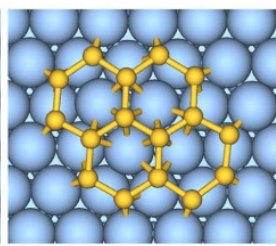

$\mathrm{Si}_{16} \mathbf{0 . 4 3}$

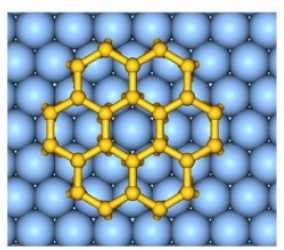

$\mathrm{Si}_{24} \mathbf{0 . 4 0}$

Figure $2 \mid$ Geometries and formation energies (eV per Si atom) of $2 \mathrm{D} \mathrm{Si}_{\mathrm{N}}$ clusters on $\operatorname{Ag}(111)$ surface.

$\mathrm{Ag}(111)$ surface. For reference, the geometries and formation energies of $\mathrm{C}_{\mathrm{N}}$ clusters on $\mathrm{Ag}(111)$ substrate are computed and shown in Supplementary Fig. S2 online. Different from the Ag-supported $\mathrm{C}_{6}$ cluster which retains its hexagon ring structure well, a hexagonal ring of $\mathrm{Si}_{6}$ placed on the $\mathrm{Ag}(111)$ surface is distorted into triangle-based configuration upon relaxation. In sharp contrast to severe deformation in vacuum, starting from $\mathrm{Si}_{10}$, the planar $\mathrm{Si}_{\mathrm{N}}$ clusters $(\mathrm{N}=10,13$, $16,19,22,24)$ formed by multiple hexagons remain stable after optimization; only $\mathrm{Si}_{13}$ (with three hexagons) undergoes moderate deformation at the periphery. Certainly, $\operatorname{Ag}(111)$ surface as the only external factor is responsible for preserving the $2 \mathrm{D}$ structure of silicene clusters, similar to the improved stability of silicon cages and nanotubes by metal stuffing ${ }^{47,48}$. This effect will be discussed in details later. Nevertheless, the well persevered six-membered rings in silicon clusters on $\operatorname{Ag}(111)$ substrate imply the existence of $\mathrm{sp}^{2}$ bonding character, which has been evidenced by experiments with STM, REELS, and ARUPS techniques ${ }^{10,13,15,20}$.

The observation of triangle-based $\mathrm{Si}_{6}$ cluster suggests a possible competition between hexagonal and triangular structural motifs for Ag-supported Si clusters. To examine the stability of the larger triangle-based silicon clusters, we have constructed one $\mathrm{Si}_{10}$ and two $\mathrm{Si}_{13}$ configurations composed by triangular networks on $\operatorname{Ag}(111)$ surface. Their atomic structures before and after optimization are shown in Supplementary Fig. S3 online. After relaxation, none of them can retain the initial triangular structures, and they all transform into some irregular shapes with higher formation energies $\left(\Delta \mathrm{E}=0.10 \mathrm{eV}\right.$ for $\mathrm{Si}_{10}, \Delta \mathrm{E}=0.13 \mathrm{eV}$ or $0.26 \mathrm{eV}$ for $\left.\mathrm{Si}_{13}\right)$ compared to the hexagon-based structures. In particular, for the $\mathrm{Si}_{13}$-t1 isomer with initial triangular structure, a hexagon would be formed spontaneously upon structural relaxation, implying the tendency of structural transformation from triangles to hexagons. The transition from triangular to hexagonal motif at $\mathrm{Si}_{10}$ may play an important role in nucleation of silicene patch, beyond which honeycombed silicene lattice can be synthesized on $\operatorname{Ag}(111)$ surface.

Formation energies of $\mathrm{Si}_{\mathrm{N}}$ clusters and interactions with $\mathbf{A g ( 1 1 1 )}$ surface. To explore the early stage of silicene nucleation, we considered

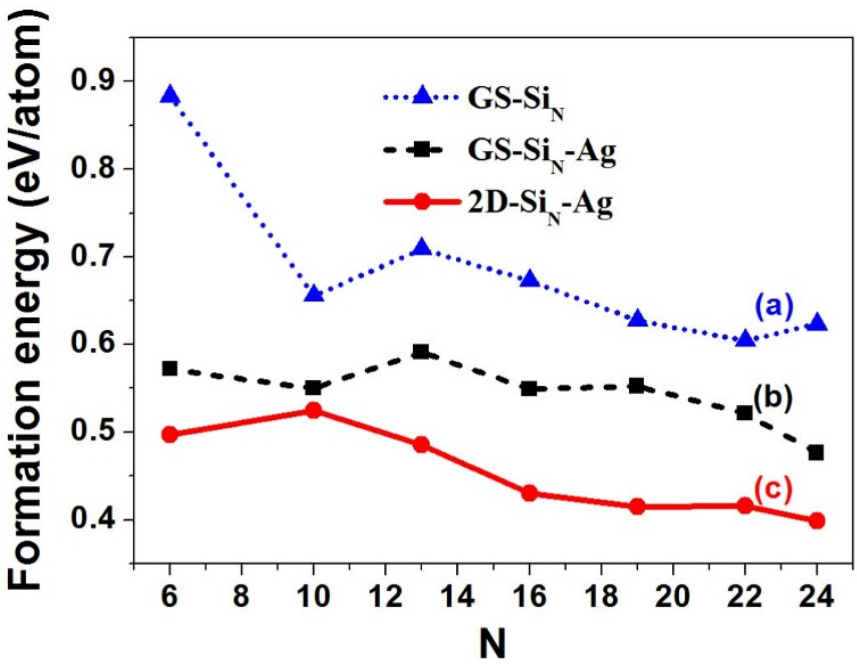

Figure 3 Formation energies of $\mathrm{Si}_{\mathrm{N}}$ clusters in vacuum and on $\mathrm{Ag}(111)$ surface: (a) freestanding $\mathrm{Si}_{\mathrm{N}}$ clusters with ground state configurations in vacuum $\left(\mathbf{G S}-\mathbf{S i}_{\mathbf{N}}\right)$; (b) direct deposition of $\mathbf{G S}-\mathbf{S i}_{\mathbf{N}}$ clusters on $\mathrm{Ag}(111)$ surface (GS-Si $\left.\mathbf{i}_{\mathbf{N}} \mathbf{A g}\right)$; (c) 2D silicene-like clusters on $\mathrm{Ag}(111)$ surface (2D-Si $\left.\mathbf{i}_{\mathrm{N}}-\mathbf{A g}\right)$.

two competitive structural motifs for $\mathrm{Si}_{\mathrm{N}}$ clusters $(\mathrm{N}=6,10,13,16$, 19, 22, 24) on $\operatorname{Ag}(111)$ substrate: (i) $2 \mathrm{D}$ silicene-like configurations as aggregates of up to seven 6MRs (Fig. 2); (ii) 3D structures that are the ground state geometries in vacuum (see Supplementary Fig. S4 online). The formation energies of these two kinds of metalsupported silicon clusters are compared in Fig. 3, along with those for freestanding clusters. When a gas-phase GS silicon cluster is deposited on the $\operatorname{Ag}(111)$ surface, its formation energies drops substantially by about $0.07 \sim 0.31 \mathrm{eV} /$ atom. This can be easily attributed to the passivation effect of metal surface, that is, the dangling bonds in the unsaturated atoms of isolated $\mathrm{Si}_{\mathrm{N}}$ clusters being passivated by the free electrons of the metal. Very interestingly, the 2D silicene-like clusters on $\operatorname{Ag}(111)$ surface are more stable than those 3D GS isomers, entirely opposite to the vacuum situation discussed above. Again, this finding can be explained by the passivation effect of $\mathrm{Ag}(111)$ surface. As shown in Supplementary Fig. S4, the metal surface only interacts with the bottom part of a 3D GS silicon cluster, leaving the top Si atoms unsaturated. On the contrary, for a $2 \mathrm{D}$ silicene cluster lying on the metal surface, all these under-coordinated $\mathrm{Si}$ atoms (with typical vertical distance of $2.2 \AA \sim 2.5 \AA$ from the Ag substrate) can be passivated. In other words, $2 \mathrm{D} \mathrm{Si}_{\mathrm{N}}$ cluster has a larger contact area with the Ag surface than the $3 \mathrm{D}$ one. As a natural extension of $2 \mathrm{D} \mathrm{Si}_{24}$ clusters with seven $6 \mathrm{MRs}$, one can speculate that an appreciably large silicene patch should be stabilized by the metal surface.

As shown in Fig. 3, the formation energy of silicon cluster increases from $\mathrm{Si}_{6}(0.50 \mathrm{eV} / \mathrm{Si}$ atom $)$ to $\mathrm{Si}_{10}(0.53 \mathrm{eV} / \mathrm{Si}$ atom $)$. After $\mathrm{Si}_{10}$, the formation energy of $\mathrm{Ag}(111)$-supported $\mathrm{Si}_{\mathrm{N}}$ cluster reduces smoothly as cluster size increases (see Fig. $3 \mathrm{~b}$ ). Thus, $\mathrm{Si}_{10}$ composed of two $6 \mathrm{MRs}$ can be considered as the nucleation size from thermodynamic point of view, which is very crucial to the growth of 2D silicene crystal. As shown in Supplementary Fig. S5a online, the diffusion barrier of silicon monomer on $\operatorname{Ag}(111)$ surface is only $0.031 \mathrm{eV}$; in other words, motion of $\mathrm{Si}$ adatoms on Ag substrate is nearly barrierless at room temperature. Thus, once overcoming the critical size of $\mathrm{Si}_{10}$, silicene patch would grow continuously until the concentration of silicon atoms from the source is not sufficient.

To gain deeper insight into the interaction mechanism between silicon clusters and silver surface, the side view of selected $2 \mathrm{D} \mathrm{Si}_{\mathrm{N}}$ clusters $(\mathrm{N}=10,13,22,24)$ are shown in Fig. 4a, along with the height difference $\Delta \mathrm{Z}$ between upper and bottom $\mathrm{Si}$ atoms for each cluster. 


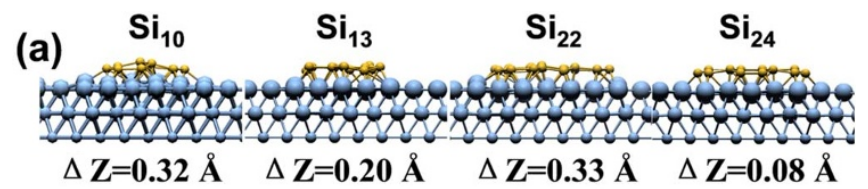

(b)
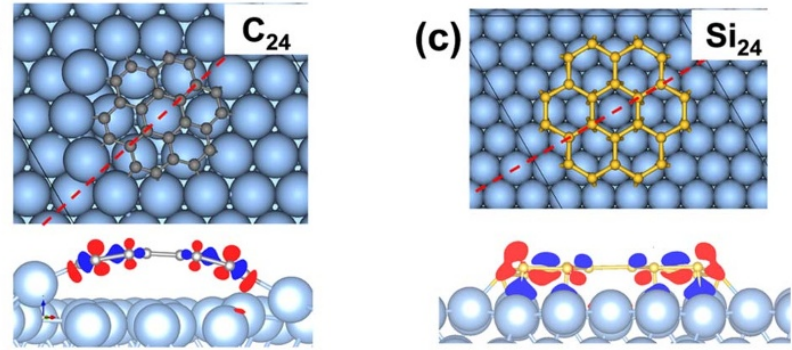

Figure 4 | Side view plots for atomic structures of (a) selected $\mathrm{Si}_{\mathrm{N}}$ clusters on $\mathrm{Ag}(111)$ surface. Charge differential densities of hexagon-based (b) $\mathrm{C}_{24}$ and (c) $\mathrm{Si}_{24}$ on $\mathrm{Ag}(111)$ surface, respectively. In (b) and (c), the cleaved planes are along the red line, and red (blue) zone loses (gains) charge.

The detailed buckling information of these $2 \mathrm{D} \mathrm{Si}_{\mathrm{N}}$ clusters is provided in the Supplementary Fig. S6 online. To explore the difference between silicene and graphene, we considered a series of 6MR-based carbon clusters on $\operatorname{Ag}(111)$ surface. Their equilibrium geometries and formation energies are shown in provided in the Supplementary Fig. S2 online. On $\mathrm{Ag}(111)$ surface, the smaller $\mathrm{C}_{10}$ and $\mathrm{C}_{13}$ clusters prefer upright standing, while the larger ones $\left(\mathrm{C}_{22}\right.$ and $\mathrm{C}_{24}$ ) form dome-like shape, similar to the previous observation of carbon nanoislands on $\operatorname{Ir}(111)$ surface $^{35}$. Different from carbon clusters, silicon clusters explored here sit more flatly on $\mathrm{Ag}(111)$ surface with slight buckling of less than $0.33 \AA$ (see Fig. 4a and Fig. S6). The geometry difference between $\mathrm{Si}_{\mathrm{N}}$ and $\mathrm{C}_{\mathrm{N}}$ clusters implies that their interactions with $\mathrm{Ag}(111)$ surface must be rather different.

As shown in Fig. 4 (b) and (c), the interactions of $\mathrm{Si}_{24}$ and $\mathrm{C}_{24}$ clusters with metal surface are visualized by charge differential density. No charge density is found between the six inner carbon atoms of $\mathrm{C}_{24}$ and $\mathrm{Ag}(111)$ surface, implying weak van der Waals (vdW) interaction rather than strong chemical bonds. Strong charge distribution between the edge of carbon cluster and $\mathrm{Ag}(111)$ surface illustrates that carbon clusters bond to metal surface mainly at the edge $e^{35}$. As for $\mathrm{Si}_{24} @ \mathrm{Ag}(111), \mathrm{Si}-\mathrm{Ag}$ charge transfer occurs both at the periphery and inner regions of $\mathrm{Si}_{24}$ cluster, suggesting that every silicon atoms interact with $\operatorname{Ag}(111)$ surface. Note that the standard DFT method used here is only able to distinguish the interactions between $\mathrm{C}$ and Si clusters with $\mathrm{Ag}(111)$ surface qualitatively, whereas accurate description of the $\mathrm{vdW}$ interaction between the dome-like carbon clusters and $\mathrm{Ag}(111)$ surface requires more elaborate methods such as DFT-D2 and vdW-DF approaches.

Previous DFT calculations predicted a slight buckling of about $0.44 \AA$ in freestanding silicene sheet ${ }^{2}$, which was also experimentally confirmed in Ag-supported silicene ML with buckling amplitude of about $1 \AA^{11-14}$. Here we also observed low buckling in 2D silicene clusters, as shown in Fig. 4a and the Supplementary Fig. S6 online. However, the buckling heights for silicene clusters $(0.2 \sim 0.33 \AA$ for $\mathrm{Si}_{10,13,22}$ and only $0.08 \AA$ for $\mathrm{Si}_{24}$ ) are less pronounced than those for the free-standing and $\mathrm{Ag}(111)$-supported silicene sheet. This phenomenon can be explained by the following picture. In vacuum, as the $\pi$ bonding is weakened, puckering of silicene sheet would introduce dehybridization. Hence, the perpendicular $\mathrm{p}_{\mathrm{z}}$ orbital combining with the s orbital is crucial to stabilize the freestanding silicene. While on $\mathrm{Ag}(111)$ slab, metal passivation helps reduce the buckling of silicene on the one side; but lattice mismatch of periodic superstructures of silicene and $\mathrm{Ag}(111)$ would introduce more seriously undulate patterns on the other side. The latter effect is dominant for

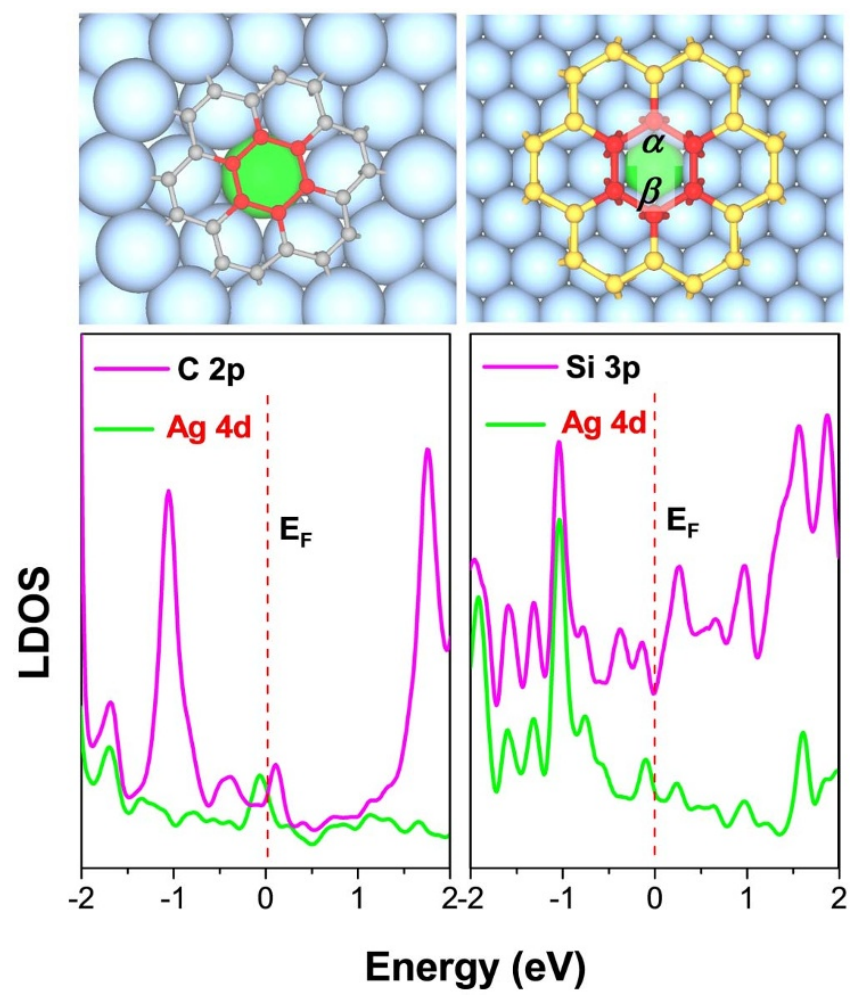

Figure $5 \mid$ Atomic structures (upper) and local density of states (LDOS, lower) of $C_{24} @ A g(111)$ (left) and $S_{24} @ A g(111)$ (right) systems. The LDOS comes from the central six carbon atoms ( $2 \mathrm{p}$ orbital) or silicon atoms ( $3 \mathrm{p}$ orbital) highlighted by red balls as well as the Ag atoms ( $4 \mathrm{~d}$ orbital) highlighted by green ball right underneath the $\mathrm{C}$ or $\mathrm{Si}$ hexagon. Two characteristic bond angles $(\alpha, \beta)$ for central $\mathrm{Si}_{6}$ hexagon are labeled: $\boldsymbol{\alpha}=120^{\circ}-121^{\circ}, \boldsymbol{\beta}=115^{\circ}-118^{\circ}$.

periodic silicene superstructures; thus high buckling of $\Delta \mathrm{Z} \sim 1 \AA$ is observed experimentally ${ }^{11-14}$. In contrast, the small silicene clusters without constraint of periodic boundary condition can accommodate the $\mathrm{Ag}(111)$ surface better since the edge atoms have more degree of freedom for adjusting the adsorption position.

Moreover, the population analysis of Mulliken charge ${ }^{49}$ of $\mathrm{Si}_{24}$ on $\mathrm{Ag}(111)$ surface is shown in the Supplementary Fig. S7 online. The on-site charges of $\mathrm{Si}$ atoms in $\mathrm{Si}_{24} @ \mathrm{Ag}(111)$ system range between $-0.13|\mathrm{e}|$ and $-0.08|\mathrm{e}|$. The slight variation of on-site charges can be associated with small height difference due to low buckling of silicene cluster (see Supplementary Fig. S6d online). Nevertheless, the nearly homogeneous charge transfer between Si atoms and Ag surface (and consequently the $\mathrm{Si}-\mathrm{Ag}$ interaction) is surely beneficial for continuous growth of silicene patch on $\mathrm{Ag}$ substrate.

To further illustrate the interaction between Si clusters and $\operatorname{Ag}(111)$ surface, we calculated the LDOS for selected atoms of $\mathrm{Si}_{24} @ \mathrm{Ag}(111)$ and $\mathrm{C}_{24} @ \mathrm{Ag}(111)$, respectively, which are displayed in Fig. 5. Clearly, the 3p states of Si atoms coincide with the Ag-4d states in the vicinity of the Fermi level, suggesting the $p_{z}$ electrons of silicon atoms hybrid with the $4 \mathrm{~d}$ electrons of $\mathrm{Ag}(111)$ surface. In contrast, there is no such correspondence between $2 p$ orbitals of carbon atoms and $4 \mathrm{~d}$ orbitals of $\mathrm{Ag}(111)$ surface, in line with the charge differential density plot (Fig. 4c) which implies vdW interaction between inner carbon hexagon and $\mathrm{Ag}$ substrate. This is because that the hybridization between $\mathrm{C} 2 \mathrm{p}$ and $\mathrm{Ag} 4 \mathrm{~d}$ states will break the delocalized $\pi$ bonds in the graphene sheet that are very stable; on the contrary, strong interaction between $\mathrm{Si} 3 \mathrm{p}$ states and Ag $4 \mathrm{~d}$ states is expected for silicene because of the reluctance of $\mathrm{Si}$ in forming $\pi$ bonds. In addition, the bond angles of the inner hexagon in $\mathrm{Si}_{24}$ split into two staggered angles: $\boldsymbol{\alpha}=120^{\circ} \sim 121^{\circ}$ and 


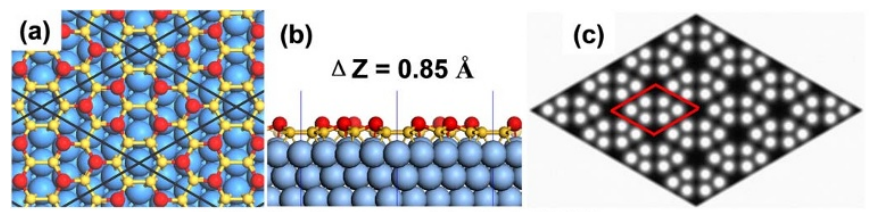

I. $(3 \times 3)$ silicene on $(4 \times 4) \mathrm{Ag}(111)$
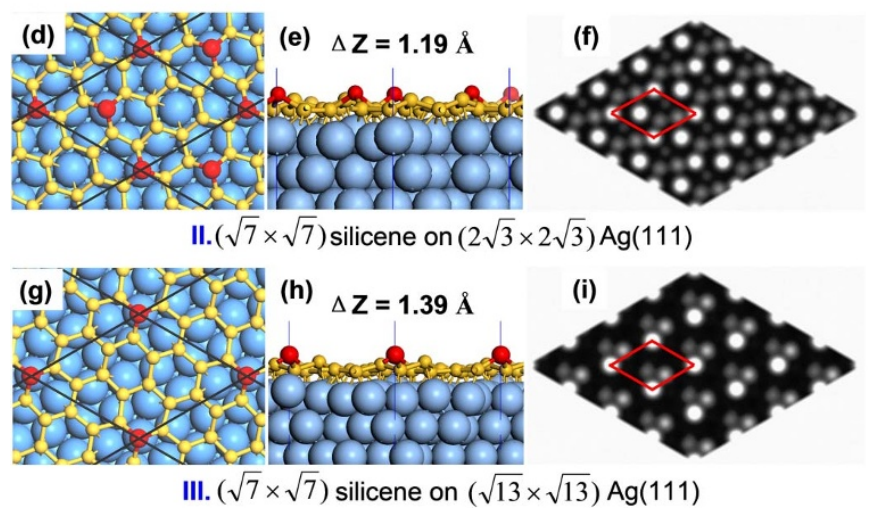

Figure 6 | Atomic structures and simulated STM images of three types of silicene@Ag(111) superstructures. (a), (d), (g) are the top views; (b), (e), (h) are the side views; $(c, f, h)$ are the STM images with a constant height of $2 \AA$ to the topmost $\mathrm{Si}$ and a bias of $-1.5 \mathrm{~V}$. The topmost Si atoms are highlighted by red balls.

$\boldsymbol{\beta}=115^{\circ} \sim 118^{\circ}$, corresponding to $\mathrm{sp}^{2}$ and $\mathrm{sp}^{2} / \mathrm{sp}^{3}$ hybridization, respectively. Similar distribution of bond angles was found in a recent study on silicene sheet on $\operatorname{Ag}(111)$ surface $^{13}$. Moreover, the LDOS of $3 p$ states of silicon exhibits a distinct pseudogap at the Fermi level like the freestanding graphene and silicene, implying that the partial $\mathrm{sp}^{2}$ characters ${ }^{10,13,20,50}$. In short, the $\mathrm{p}_{\mathrm{z}}$ orbital of Si interacts substantially with Ag substrate while $\mathrm{sp}^{2}$ hybridization still exists in these 2D silicene-like clusters on metal. Different from graphene with mainly $\mathrm{sp}^{2}$ states, the partially $\mathrm{sp}^{2}$-hybridization characteristics in $\mathrm{Ag}(111)$-supported silicene might lead to some novel applications.

Silicene monolayer superstructures on $\operatorname{Ag}(111)$ surface. To further understand the interaction between silicene and $\operatorname{Ag}(111)$ surface and to directly compare with available experiments, we considered three types of co-periodic silicene@Ag(111) superstructures: (I) $(3 \times 3)$ silicene on $(4 \times 4) \operatorname{Ag}(111)$ surface (usually named $\operatorname{Ag}(111)-(1 \times 1)$ surface or $(4 \times 4)$ silicene $\left.{ }^{11-14}\right)$; (II) $(\sqrt{7} \times \sqrt{7})$ silicene on $(2 \sqrt{3} \times$ $2 \sqrt{3}) \operatorname{Ag}(111)$ surface ${ }^{14}$; (III) $(\sqrt{7} \times \sqrt{7})$ silicene on $(\sqrt{13} \times \sqrt{13})$ $\operatorname{Ag}(111)$ surface $^{11}$. The atomic structures as well as the simulated STM images are shown in Fig. 6. The detailed structural information such as the supercell dimension, mismatch, bond length, are summarized in the Supplementary Table S8 online.

As displayed in Fig. 6, due to passivation of metal surface and lattice mismatch, the buckling behavior of $\mathrm{Ag}(111)$-supported silicene sheet is quite different from the freestanding ones. In the frequently found superstructure of type I, there are only $1 / 3$ of Si atoms at the top (highlighted by red balls), resulting a wheel-shaped double ring STM pattern (Fig. 6c) composed by six spots on the internal ring and twelve spots on the external ring, in excellent agreement with experimental images ${ }^{11-13}$. Within the silicene@Ag(111) supercell of type II, there are only two $\mathrm{Si}$ atoms (1/7 of $\mathrm{Si}$ atoms) on the top sites, resulting a honeycombed STM image (Fig. 6f); however, the nearest distance between two spots is about $5.76 \AA^{14}$. In the superstructure of type III, there is only one topmost $\mathrm{Si}$ atom per cell corresponding to the lightest spot in STM image (Fig. 6i). The present theoretical result for type III can be related to recent experimental STM image by Lin et al. ${ }^{11}$, in which they observed one spot per rhombus supercell of $10.4 \AA$ in dimension.
The heights of buckling in these three types of silicene superstructure are $0.85 \AA, 1.19 \AA$ and $1.39 \AA$, respectively. Considering the corresponding lattice mismatches of $-0.86 \%,+2.73 \%$ and -1.24 $\%$, no clear correlation is found between the buckling amplitude and the lattice mismatch. As listed in the Supplementary Table S8 online, the Si-Si bond lengths of these three types of superstructures range between $2.285 \AA$ and $2.436 \AA$, in accordance with previous theoretical calculations and experimental data ${ }^{11-14}$. The average $\mathrm{Si}$-Si bond length of $\mathrm{Ag}(111)$-supported silicene ML superstructures are $2.351 \AA$ for $(4 \times 4), 2.322 \AA$ for $(2 \sqrt{3} \times 2 \sqrt{3})$ and $2.357 \AA$ for $(\sqrt{13} \times \sqrt{13})$, respectively. Interestingly, the variation of $\mathrm{Si}-\mathrm{Si}$ bond length is roughly proportional to the mismatch between silicene superstructure and $\operatorname{Ag}(111)$ surface.

Thermal stability of silicene monolayers on $\mathrm{Ag}(111)$ and $\mathrm{Rh}(111)$ surfaces. The above analyses indicate that the $2 \mathrm{D}$ silicene-like clusters are stabilized by $\operatorname{Ag}(111)$ surface due to passivation of the unsaturated edge $\mathrm{Si}$ atoms and $\mathrm{p}$-d hybridization between inner $\mathrm{Si}$ atoms and Ag substrate. Due to the lattice mismatch between $\mathrm{Ag}(111)$ and silicene lattice as well as the energy difference between different adsorption sites on metal surface, deposition of large-scale silicene sheet on the metal substrate would introduce extra local tension on silicene. At finite temperature, thermal perturbation would trigger some structural defects associated with such local tension and thus disturb the geometry integrity of silicene sheet. This effect must be considered during the fabrication and processing of the silicene-based materials and devices. Previously, phonon calculations have been conducted to assess the stability of the freestanding silicene $\mathrm{ML}^{2}$; but such simulation is unable to examine the finite-temperature behavior and the thermal stability.

To assess the thermal stability of silicene on metal substrates, we preformed AIMD simulation on the type I silicene@Ag(111) superstructure as a representative. The initial geometry of silicene ML on $\operatorname{Ag}(111)$ was fully optimized (see Fig. 6a,b and Fig. 7a). AIMD simulations were then carried out at $500 \mathrm{~K}$ (experimental growth temperature) for more than $7.5 \mathrm{ps}$. Interestingly, the silicene monolayer is found to be very stable on $\operatorname{Ag}(111)$ surface. At $500 \mathrm{~K}$, no topological defect was ever generated during the entire simulation time of 7.5 ps (Fig. 7b), confirming its high thermal stability on $\operatorname{Ag}(111)$ surface. This can be attributed to the passivation effect as well as low local tension of silicene network on the metal surface. As shown in Supplementary Fig. S6 online, the local energy difference of silicon monomer on different sites of $\operatorname{Ag}(111)$ surface is lower than $0.031 \mathrm{eV}$, implying a very small local tension.

To understand the role of $\operatorname{Ag}(111)$ surface, the $\mathrm{Rh}(111)$ surface, which has relatively stronger interaction with $\mathrm{Si}$, was assumed as substrate of silicene monolayer. A silicene@Rh(111) superstructure, including $(4 \times 4)$ silicene cells and $(6 \times 6) \mathrm{Rh}(111)$ cells with $3.7 \%$ mismatch, was constructed. Upon DFT relaxation, silicene on $\mathrm{Rh}$ (111) surface can retain its honeycomb lattice well (Fig. 8a). Comparative AIMD simulation at $500 \mathrm{~K}$ was then carried for this silicene@ $\mathrm{Rh}(111)$ superstructure up to $2.3 \mathrm{ps}$. In sharp contrast to that on $\operatorname{Ag}(111)$ surface, the silicene lattice on $\mathrm{Rh}(111)$ surface start collapsing via breaking up two $\mathrm{Si}$-Si bonds after only 0.7 ps simulation time (Fig. 8b). Once such a defect is created, silicene monolayer became more and more unstable on $\mathrm{Rh}(111)$ surface. After the entire duration of $2.3 \mathrm{ps}$, an amorphous Si-Rh interface is formed (Fig. 8c).

The thermal instability of silicene ML on $\mathrm{Rh}(111)$ may be interpreted by the larger local energy difference of $\mathrm{Si}$ atom on $\mathrm{Rh}$ surface. As shown in Supplementary Fig. S5b online, the diffusion barrier of silicon monomer on $\mathrm{Rh}(111)$ surface is $0.306 \mathrm{eV}$, about ten times than that on $\operatorname{Ag}(111)$ surface. However, the co-periodic silicene@ $\mathrm{Rh}(111)$ supercell considered here has a large lattice mismatch of $3.7 \%$, which might also account for the instability of silicene ML. To exclude this factor, we further adopted a $(5 \times 5)$ silicene on $(7 \times 7)$ $\mathrm{Ag}(111)$ surface (Fig. 7c) with $3.8 \%$ lattice mismatch for AIMD 
$(3 \times 3)$ silicene on $(4 \times 4) \mathrm{Ag}(111), 0.8 \%$ mismatch

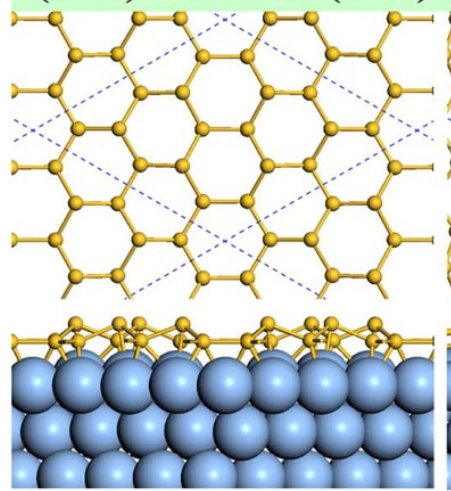

(a) $0.0 \mathrm{ps}$
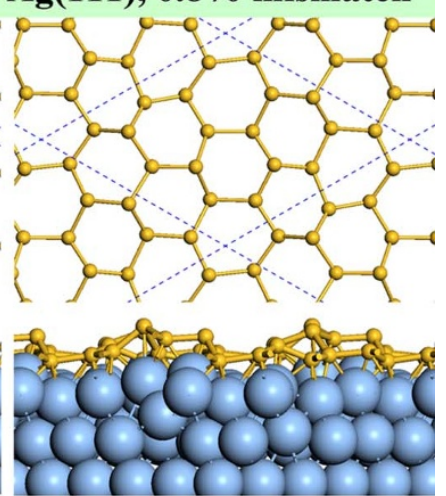

(b) $7.5 \mathrm{ps}, 500 \mathrm{~K}$

\section{$(5 \times 5)$ silicene on $(7 \times 7) \mathrm{Ag}(111), 3.8 \%$ mismatch}
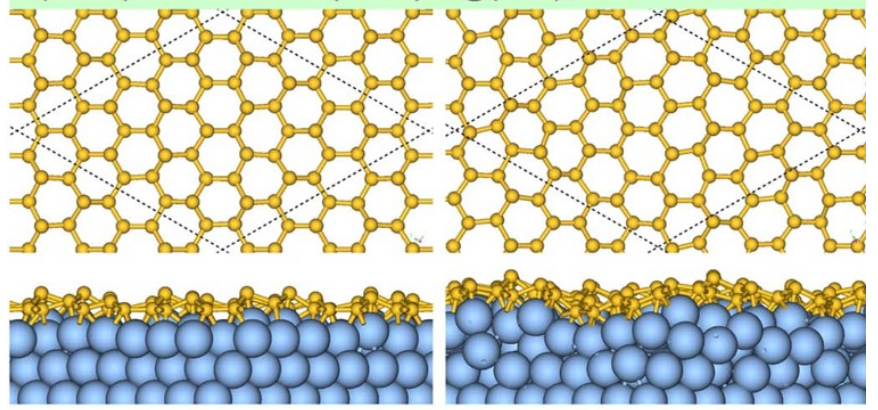

(c) $0.0 \mathrm{ps}$

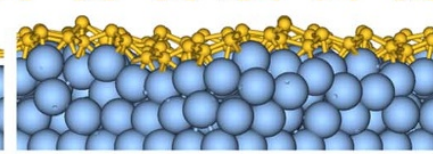

(d) $5.6 \mathrm{ps}, 500 \mathrm{~K}$

Figure 7 | Snapshots from AIMD simulation of silicene monolayers on $\operatorname{Ag}(111)$ at $500 \mathrm{~K}$. (a) initial configuration and (b) structure after 7.5 ps for $(3 \times 3)$ silicene on $(4 \times 4) \operatorname{Ag}(111)$ surface (i.e., type I defined above); (c) initial configuration and (d) structure after 5.6 ps for $(5 \times 5)$ silicene on $(7 \times 7) \mathrm{Ag}(111)$ surface with $3.8 \%$ lattice mismatch. For each graph, Ag atoms are not shown in top view.

simulation. During the simulation time (5.6 ps), silicene lattice is well preserved on $\operatorname{Ag}(111)$ surface and not even a defect is produced at $500 \mathrm{~K}$. Interestingly, even at a higher temperature of $900 \mathrm{~K}$, the topological structure of silicene lattice is still retained for 5 ps (see Supplementary Fig. S9 online for details). Thus we can conclude that the instability of silicene on $\mathrm{Rh}(111)$ surface is mainly originated from high local energy difference. These results suggest that the local energy difference of silicon atoms on surface might be a key factor to justify whether a metal substrate is suitable for epitaxial growth of silicene.
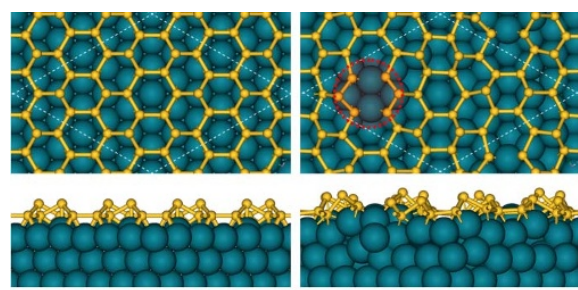

(a) Rh $0.0 \mathrm{ps}$

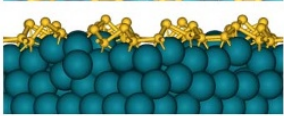

(b) Rh $0.7 \mathrm{ps;} \mathrm{500K}$

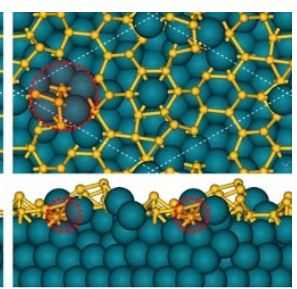

(c) Rh 2.3 ps; 500K
Figure 8 Snapshots from AIMD simulation of silicene on $\mathrm{Rh}(111)$ at $500 \mathrm{~K}$. (a) initial structure; (b) structure at 0.7 ps with two Si-Si bonds broken; (c) structure after 2.3 ps, showing an amorphous Si-Rh interface. The important local disruptions of silicene 2D lattice are labeled by red dash rings for guide of eyes.

\section{Discussion}

In summary, using $a b$ initio calculations, the structures and stabilities of $\mathrm{Si}_{\mathrm{N}}$ clusters $(\mathrm{N} \leq 24)$ in vacuum and on $\mathrm{Ag}(111)$ surface are investigated and compared with those of $\mathrm{C}_{\mathrm{N}}$ clusters. Planar configurations based on aggregates of hexagons are highly unstable for the isolated silicon clusters in vacuum and will be severely reconstructed to 3D distorted structures spontaneously. However, due to passivation effect of metal surface, these $2 \mathrm{D}$ silicon clusters as embryo of silicene patch can be stabilized by $\operatorname{Ag}(111)$ substrate. No dome shape is found for $\mathrm{Ag}(111)$-supported $\mathrm{Si}_{\mathrm{N}}$ clusters, in contrast to graphene nanoclusters on TM surfaces. Analysis of electronic structures reveals significant $\mathrm{p}-\mathrm{d}$ hybridization between $\mathrm{Si}$ and $\mathrm{Ag}$ surface, and $\mathrm{sp}^{2}$ characteristics in silicone clusters. Three types of silicene superstructures on $\operatorname{Ag}(111)$ surface are investigated and the simulated STM images agree well with available experimental observations. Compared to the buckling of about $0.44 \AA$ in freestanding silicene sheet, the buckling heights in 2D silicene clusters $(0.08 \sim$ $0.33 \AA$ ) on $\operatorname{Ag}(111)$ substrate are smaller and those in $\mathrm{Ag}(111)$-supported silicene sheet $(0.85 \sim 1.39 \AA)$ are larger. AIMD simulations on two kinds of silicene@Ag(111) superstructures and comparing with that on $\mathrm{Rh}(111)$ surface confirm excellent thermal stability of silicene monolayer on Ag surface, which can be attributed to the passivation effect by Ag and low local energy difference of silicon adatoms on different sites of $\operatorname{Ag}(111)$ surface. These theoretical results not only are very helpful for understanding the atomic structures, the initial growth behaviors, and the interaction of silicene on $\operatorname{Ag}(111)$ surface, but also provide some useful guidelines for finding new appropriate substrate for epitaxial growth of silicene beyond Ag surface.

\section{Methods}

$A b$ initio calculations were performed by using density functional theory (DFT) and plane wave basis, as implemented in the Vienna A $b$-initio Simulation Package (VASP) ${ }^{51,52}$. Generalized gradient approximation (GGA) with the RPBE functional was adopted to describe the exchange-correlation interaction, which was elaborately developed for the calculations of surface systems ${ }^{53}$. The core electrons were described by the projected augmented wave (PAW) method $^{54}$. The kinetic energy cutoff of $400 \mathrm{eV}$ for the plane wave basis was used.

In this study, we chose the $\mathrm{Ag}(111)$ surface as substrate since it was the major metal surface for growing large-scale silicene sheet till now ${ }^{11-14}$. The $\mathrm{Ag}(111)$ surface was modeled by a three-layer slab model within periodic boundary condition, which was cleaved from bulk fcc silver solid with the experimental lattice constant of $2.89 \AA$. The validity of this three-layer slab model was assessed by our previous studies ${ }^{37,39}$ as well as our test calculations on a five-layer slab. To avoid the interactions between adjacent periodic images, a large $(7 \times 7)$ supercell with dimension of $20.22 \AA \times 20.22 \AA$ was used for the slab model, while the supercell dimension perpendicular to the $\mathrm{Ag}(111)$ surface was chosen as large as $30 \AA$. With fixed supercell parameters, the three-layer slab model was further relaxed with the fixed bottom layer to mimic the semi-infinite solid. Then, different sized $\mathrm{Si}_{\mathrm{N}}$ and $\mathrm{C}_{\mathrm{N}}$ clusters (up to $\mathrm{N}=24$ ) were placed on the optimized $(7 \times 7)$ slab model of $\mathrm{Ag}(111)$ surface and the entire cluster-substrate systems were fully relaxed (also with the bottom layer of Ag atoms fixed). During the geometry optimization, a $(2 \times 2) \mathrm{k}$-point mesh including the $\boldsymbol{\Gamma}$ point was used to sample the reciprocal space due to the large supercell. For LDOS calculation, the $\mathbf{k}$ point mesh was increased to $(7 \times 7)$ in order to obtain more accurate information on the electronic structures. To simulate the diffusion behavior of $\mathrm{Si}$ atom on $\mathrm{Ag}(111)$ and $\mathrm{Rh}(111)$ surface, the climbing image nudged elastic band (cNEB) method with convergence criterion of $0.02 \mathrm{eV} / \AA$ for force was used to find the diffusion path $\mathrm{h}^{55}$.

The structure of Silicene@Ag(111) were investigate by three selected silicene superstructures on $\operatorname{Ag}(111)$ surface by compressing silicene lattice slightly to fit the metal surface ${ }^{56}$. The k-point meshes are divided into smaller than $0.03 \AA^{-1}$ for silicene metal superstructures. The STM images of silicene superstructures were simulated by using the Tersoff-Hamann approximation ${ }^{57}$ with a constant height of $2 \AA$ above the topmost $\mathrm{Si}$ atoms. To examine the thermal stability of silicene ML on $\mathrm{Ag}(111)$ surfaces, $a b$ initio molecular dynamics simulation within the NVT ensemble ${ }^{58}$ were performed using VASP. The system for AIMD simulation refers to the most observed superstructure composed of $(3 \times 3)$ silicene cells and $(4 \times 4) \operatorname{Ag}(111)$ cells with only $-0.86 \%$ mismatch. AIMD simulation at $500 \mathrm{~K}$ (which is the typical experimental growth temperature) lasted for 7.5 ps. To shed light on the role of $\mathrm{Ag}(111)$ surface, a comparative AIMD simulation of silicene ML on a strongly interacting $\mathrm{Rh}(111)$ surface was carried out and the simulation supercell included $(4 \times 4)$ silicene cell on $(6 \times 6)$ cell of $\mathrm{Rh}(111)$ surface $(3.7 \%$ mismatch). Furthermore, to distinguish whether the large mismatch or $\mathrm{Rh}(111)$ 's self caused worse thermal stable of silicene, a larger silicene@Ag(111) co-periodic superstructure, including $(5 \times 5)$ silicene cells on $(7 \times 7)$ $\mathrm{Ag}(111)$ cells (3.8\% mismatch) was used to compare that of $3.7 \%$ mismatched silicene@Rh(111) superstructure upon more than 5.0 ps AIMD simulation. The time step for all AIMD simulations was $1.0 \mathrm{fs}$. In all situations, the convergence criterion of 
total energy for self-consistent field (SCF) calculations was set as $10^{-4} \mathrm{eV}$ and the convergence criterion of $0.02 \mathrm{eV} / \AA$ for force was used during geometry optimization.

1. Guzmán-Verri, G. G. \& Lew Yan Voon, L. C. Electronic structure of silicon-based nanostructures. Phys. Rev. B 76, 075131 (2007).

2. Cahangirov, S., Topsakal, M., Aktürk, E., Şahin, H. \& Ciraci, S. Two- and OneDimensional Honeycomb Structures of Silicon and Germanium. Phys. Rev. Lett. 102, 236804 (2009).

3. Morishita, T., Nishio, K. \& Mikami, M. Formation of single- and double-layer silicon in slit pores. Phys. Rev. B 77, 081401 (2008).

4. Takeda, K. \& Shiraishi, K. Theoretical possibility of stage corrugation in $\mathrm{Si}$ and $\mathrm{Ge}$ analogs of graphite. Phys. Rev. B 50, 14916-14922 (1994).

5. Yang, X. \& Ni, J. Electronic properties of single-walled silicon nanotubes compared to carbon nanotubes. Phys. Rev. B 72, 195426 (2005).

6. Léandri, C., Oughaddou, H., Aufray, B., Gay, J. M., Le Lay, G., Ranguis, A. \& Garreau, Y. Growth of Si nanostructures on $\operatorname{Ag}(00$ 1). Surf. Sci. 601, 262-267 (2007).

7. Aufray, B., Kara, A., Vizzini, S., Oughaddou, H., Leandri, C., Ealet, B. \& Le Lay, G. Graphene-like silicon nanoribbons on $\mathrm{Ag}(110)$ : A possible formation of silicene. Appl. Phys. Lett. 96, 183102-183103 (2010).

8. De Padova, P., Quaresima, C., Ottaviani, C., Sheverdyaeva, P. M., Moras, P., Carbone, C., Topwal, D., Olivieri, B., Kara, A., Oughaddou, H., Aufray, B. \& Le Lay, G. Evidence of graphene-like electronic signature in silicene nanoribbons. Appl. Phys. Lett. 96, 261905-261903 (2010).

9. De Padova, P., Quaresima, C., Olivieri, B., Perfetti, P. \& Le Lay, G. Strong resistance of silicene nanoribbons towards oxidation. J. Phys. D: Appl. Phys. 44, 312001 (2011).

10. De Padova, P., Quaresima, C., Olivieri, B., Perfetti, P. \& Le Lay, G. sp $^{2}$ like hybridization of silicon valence orbitals in silicene nanoribbons. Appl. Phys. Lett. 98, 081909-081903 (2011).

11. Lin, C.-L., Arafune, R., Kawahara, K., Tsukahara, N., Minamitani, E., Kim, Y., Takagi, N. \& Kawai, M. Structure of Silicene Grown on $\operatorname{Ag}(111)$. Appl. Phys. Express 5, 045802 (2012)

12. Feng, B., Ding, Z., Meng, S., Yao, Y., He, X., Cheng, P., Chen, L. \& Wu, K. Evidence of silicene in honeycomb structures of silicon on $\mathrm{Ag}(111)$. Nano Lett. 12, 3507-3511 (2012).

13. Vogt, P., De Padova, P., Quaresima, C., Avila, J., Frantzeskakis, E., Asensio, M. C., Resta, A., Ealet, B. \& Le Lay, G. Silicene: Compelling Experimental Evidence for Graphenelike Two-Dimensional Silicon. Phys. Rev. Lett. 108, 155501 (2012).

14. Jamgotchian, H., Colignon, Y., Hamzaoui, N., Ealet, B., Hoarau, J. Y., Aufray, B. \& Bibérian, J. P. Growth of silicene layers on $\mathrm{Ag}(111)$ : unexpected effect of the substrate temperature. J. Phys. Condens. Matter 24, 172001 (2012).

15. Fleurence, A., Friedlein, R., Ozaki, T., Kawai, H., Wang, Y. \& Yamada-Takamura, Y. Experimental Evidence for Epitaxial Silicene on Diboride Thin Films. Phys. Rev. Lett. (2012).

16. Ding, Y. \& Ni, J. Electronic structures of silicon nanoribbons. Appl. Phys. Lett. 95 , 083115-083113 (2009).

17. Lebègue, S. \& Eriksson, O. Electronic structure of two-dimensional crystals from ab initio theory. Phys. Rev. B 79, 115409 (2009).

18. Şahin, H., Cahangirov, S., Topsakal, M., Bekaroglu, E., Akturk, E., Senger, R. T. \& Ciraci, S. Monolayer honeycomb structures of group-IV elements and III-V binary compounds: First-principles calculations. Phys. Rev. B 80, 155453 (2009).

19. Liu, C.-C., Jiang, H. \& Yao, Y. Low-energy effective Hamiltonian involving spinorbit coupling in silicene and two-dimensional germanium and tin. Phys. Rev. B 84, 195430 (2011).

20. Chen, L., Liu, C.-C., Feng, B., He, X., Cheng, P., Ding, Z., Meng, S., Yao, Y. \& Wu, K. Evidence for Dirac Fermions in a Honeycomb Lattice Based on Silicon. Phys. Rev. Lett. 109, 056804 (2012).

21. Liu, C.-C., Feng, W. \& Yao, Y. Quantum Spin Hall Effect in Silicene and Two-Dimensional Germanium. Phys. Rev. Lett. 107, 076802 (2011)

22. Ni, Z., Liu, Q., Tang, K., Zheng, J., Zhou, J., Qin, R., Gao, Z., Yu, D. \& Lu, J. Tunable Bandgap in Silicene and Germanene. Nano Lett. 12, 113-118 (2011)

23. Drummond, N. D., Zólyomi, V. \& Fal'ko, V. I. Electrically tunable band gap in silicene. Phys. Rev. B 85, 075423 (2012).

24. Kamal, C. Controlling Band Gap in Silicene Monolayer Using External Electric Field. arXiv:1202.2636v1[cond-mat.mes-hall] (2012).

25. O’Hare, A., Kusmartsev, F. V. \& Kugel, K. I. A Stable "Flat” Form of Two-Dimensional Crystals: Could Graphene, Silicene, Germanene Be Minigap Semiconductors? Nano Lett. 12, 1045-1052 (2012).

26. Osborn, T. H., Farajian, A. A., Pupysheva, O. V., Aga, R. S. \& Lew Yan Voon, L. C. $\mathrm{Ab}$ initio simulations of silicene hydrogenation. Chem. Phys. Lett. 511, 101-105 (2011).

27. Houssa, M., Scalise, E., Sankaran, K., Pourtois, G., Afanas'ev, V. V. \& Stesmans, A. Electronic properties of hydrogenated silicene and germanene. Appl. Phys. Lett. 98, 223107-223103 (2011)

28. Wang, X.-Q., Li, H.-D. \& Wang, J.-T. Induced ferromagnetism in one-side semihydrogenated silicene and germanene. Phys. Chem. Chem. Phys. 14, 3031-3036 (2012).

29. Ding, Y. \& Wang, Y. Electronic structures of silicene fluoride and hydride. Appl. Phys. Lett. 100, 083102-083104 (2012).
30. Gao, N., Zheng, W. T. \& Jiang, Q. Density functional theory calculations for two-dimensional silicene with halogen functionalization.

Phys. Chem. Chem. Phys. 14, 257-261 (2012).

31. Kara, A., Enriquez, H., Seitsonen, A. P., Lew Yan Voon, L. C., Vizzini, S., Aufray, B. \& Oughaddou, H. A review on silicene - New candidate for electronics. Surf. Sci. Rep. 67, 1-18 (2012).

32. Jose, D. \& Datta, A. Structures and electronic properties of silicene clusters: a promising material for FET and hydrogen storage. Phys. Chem. Chem. Phys. 13, 7304-7311 (2011).

33. Gao, J., Yip, J., Zhao, J., Yakobson, B. I. \& Ding, F. Graphene Nucleation on Transition Metal Surface: Structure Transformation and Role of the Metal Step Edge. J. Am. Chem. Soc. 133, 5009-5015 (2011).

34. Gao, J., Yuan, Q., Hu, H., Zhao, J. \& Ding, F. Formation of Carbon Clusters in the Initial Stage of Chemical Vapor Deposition Graphene Growth on Ni(111) Surface. J. Phys. Chem. C 115, 17695-17703 (2011).

35. Lacovig, P., Pozzo, M., Alfe, D., Vilmercati, P., Baraldi, A. \& Lizzit, S. Growth of Dome-Shaped Carbon Nanoislands on $\operatorname{Ir}(111)$ : The Intermediate between Carbidic Clusters and Quasi-Free-Standing Graphene. Phys. Rev. Lett. 103, 166101 (2009)

36. Wu, P., Jiang, H., Zhang, W., Li, Z., Hou, Z. \& Yang, J. Lattice Mismatch Induced Nonlinear Growth of Graphene. J. Am. Chem. Soc. 134, 6045-6051 (2012).

37. Yuan, Q., Gao, J., Shu, H., Zhao, J., Chen, X. \& Ding, F. Magic Carbon Clusters in the Chemical Vapor Deposition Growth of Graphene. J. Am. Chem. Soc. 134, 2970-2975 (2011).

38. Gao, J., Zhao, J. \& Ding, F. Transition Metal Surface Passivation Induced Graphene Edge Reconstruction. J. Am. Chem. Soc. 134, 6204-6209 (2012).

39. Cheng, D., Barcaro, G., Charlier, J.-C., Hou, M. \& Fortunelli, A. Homogeneous Nucleation of Graphitic Nanostructures from Carbon Chains on Ni(111). J. Phys. Chem. C 115, 10537-10543 (2011).

40. Meng, L., Sun, Q., Wang, J. \& Ding, F. Molecular Dynamics Simulation of Chemical Vapor Deposition Graphene Growth on Ni (111) Surface. J. Phys. Chem. C 116, 6097-6102 (2012).

41. Amara, H., Bichara, C. \& Ducastelle, F. Formation of carbon nanostructures on nickel surfaces: A tight-binding grand canonical Monte Carlo study. Phys. Rev. B 73, 113404 (2006).

42. Wu, P., Zhang, W., Li, Z., Yang, J. \& Hou, J. G. Communication: Coalescence of carbon atoms on $\mathrm{Cu}(111)$ surface: Emergence of a stable bridging-metal structure motif. J. Chem. Phys. 133, 071101-071104 (2010).

43. Zhu, X. \& Zeng, X. C. Structures and stabilities of small silicon clusters: Ab initio molecular-orbital calculations of $\mathrm{Si}_{7}-\mathrm{Si}_{11}$. J. Chem. Phys. 118, 3558-3570 (2003)

44. Zhu, X. L., Zeng, X. C., Lei, Y. A. \& Pan, B. Structures and stability of medium silicon clusters. II. Ab initio molecular orbital calculations of $\mathrm{Si}_{12}-\mathrm{Si}_{20}$. J. Chem. Phys. 120, 8985-8995 (2004).

45. Yoo, S. \& Zeng, X. C. Structures and relative stability of medium-sized silicon clusters. IV. Motif-based low-lying clusters $\mathrm{Si}_{21}-\mathrm{Si}_{30}$. J. Chem. Phys. 124, 054304-054306 (2006).

46. Kosimov, D. P., Dzhurakhalov, A. A. \& Peeters, F. M. Carbon clusters: From ring structures to nanographene. Phys. Rev. B 81, 195414 (2010).

47. Kumar, V. Nanosilicon. (Elsevier Science, 2007).

48. Wang, J., Zhao, J., Ma, L., Wang, G. \& King, R. B. Stability and magnetic properties of Fe encapsulating in silicon nanotubes. Nanotechnology 18, 235705 (2007).

49. Mulliken, R. S. Electronic Population Analysis on LCAO-MO Molecular Wave Functions. I. J. Chem. Phys. 23, 1833-1840 (1955).

50. Kara, A., Vizzini, S., Leandri, C., Ealet, B., Oughaddou, H., Bernard, A. \& Le Lay, G. Silicon nano-ribbons on $\mathrm{Ag}(110)$ : a computational investigation. J. Phys Condens. Matter 22, 045004 (2010).

51. Kresse, G. \& Furthmüller, J. Efficient iterative schemes for ab initio total-energy calculations using a plane-wave basis set. Phys. Rev. B 54, 11169-11186 (1996).

52. Kresse, G. \& Furthmüller, J. Efficiency of ab-initio total energy calculations for metals and semiconductors using a plane-wave basis set. Comput. Mater. Sci. 6, 15-50 (1996)

53. Hammer, B., Hansen, L. B. \& Nørskov, J. K. Improved adsorption energetics within density-functional theory using revised Perdew-Burke-Ernzerhof functionals. Phys. Rev. B 59, 7413-7421 (1999).

54. Blöchl, P. E. Projector augmented-wave method. Phys. Rev. B 50, 17953-17979 (1994)

55. Henkelman, G., Uberuaga, B. P. \& Jónsson, H. A climbing image nudged elastic band method for finding saddle points and minimum energy paths. J. Chem. Phys. 113, 9901-9904 (2000)

56. Giovannetti, G., Khomyakov, P. A., Brocks, G., Karpan, V. M., van den Brink, J. \& Kelly, P. J. Doping Graphene with Metal Contacts. Phys. Rev. Lett. 101, 026803 (2008).

57. Tersoff, J. \& Hamann, D. R. Theory and Application for the Scanning Tunneling Microscope. Phys. Rev. Lett. 50, 1998-2001 (1983).

58. Windiks, R. \& Delley, B. Massive thermostatting in isothermal density functional molecular dynamics simulations. J. Chem. Phys. 119, 2481-2487 (2003).

\section{Acknowledgment}

This work was supported by the National Natural Science Foundation of China (11134005), Program for Changjiang Scholars and Innovative Research Team in University of China, 
Fundamental Research Funds for the Central Universities of China (No. DUT12YQ05), and Program for SCI@guoshi of CETV.

\section{Author contributions}

Junfeng Gao and Jijun Zhao wrote the manuscript and prepared the figures. Junfeng Gao did the calculations. All authors reviewed the manuscript.

\section{Additional information}

Supplementary information accompanies this paper at http://www.nature.com/ scientificreports
Competing financial interests: The authors declare no competing financial interests. License: This work is licensed under a Creative Commons Attribution-NonCommercialNoDerivs 3.0 Unported License. To view a copy of this license, visit http:// creativecommons.org/licenses/by-nc-nd/3.0/

How to cite this article: Gao, J. \& Zhao, J. Initial geometries, interaction mechanism and high stability of silicene on Ag(111) surface. Sci. Rep. 2, 861; DOI:10.1038/srep00861 (2012). 